\title{
Research on Texture based on the Method of Image Retrieval
}

\author{
Lei Ao \\ Network engineering teaching and research section \\ City institute, Dalian university of technology, Dalian, China \\ 154001958@qq.com
}

Keywords: Texture, Co-occurrence matrix, Roughness, Contrast, Directionality.

\begin{abstract}
The texture in digital images is the spatial correlation of the gray or color of adjacent pixels, or the visual representation of the change of image gray level and color space position, which can be expressed as edges, shapes, stripes, color blocks and so on. Therefore, to extract the texture features by the method of mathematics or information theory, the image grayscale or color space overall or each side with digital feature representation, then using texture analysis method to detect the texture element texton and access to relevant information distribution arrangement.
\end{abstract}

\section{Introduction}

The color is an important feature of image visual perception, but due to the neglect of other features, and the comprehensive information of image compression only to a color space, so the image semantic information representation ability is insufficient, easy to appear the color feature similar semantically unrelated situations. According to the human visual characteristics, in certain areas of the image, a certain color occupies a large proportion, people only pay attention to their visual features in the visual, and do not pay attention to their color characteristics. In addition, for two images, only the color comparison is relatively close, but the semantic information of the two images is completely different. If the texture feature of the image is introduced, the distance between the two images will be widened so that the image can be retrieved more accurately..

\section{Co-Occurrence Matrix Method}

Traditional statistical analysis methods began in the early 1970s, and Haralickn proposed a co-occurrence matrix method based on two order grey scale statistical features ${ }^{[1]}$.

Take any point in the image $(x, y)$ and deviate from its other point $(x+a, y+b)$, and set the gray value of this point to be $(\mathrm{G} 1, \mathrm{G} 2)$. Orders (x,y) move on the entire picture, and then get a variety of $(G 1, G 2)$ values, set the gray value of the series is $k$, then $(G 1, G 2)$ combination of a total of k2. For the whole picture, the statistics for each $(G 1, G 2)$ value of the number of occurrences, and then arranged in a matrix, then (G1, G2) the total number of them will appear normalized to the rate of $P$ (GL, G2), the matrix is called joint probability matrix, also called co-occurrence matrix.

Through the statistics of the elements in co-occurrence matrix from different angles, four features are used to represent the texture features ${ }^{[2]:}$

A. contrast (the moment of inertia of the main diagonal)

$$
\mathrm{CON}=\sum \sum(\mathrm{h}-\mathrm{k})^{2} \mathrm{mhk}
$$

For coarse textures, the corresponding CON values are smaller because the values of $\mathrm{m}$ are more concentrated near the main diagonal, and the value is less at this time (h-k). In contrast, for fine textures, the corresponding CON values are larger.

B. energy (angular two moment)

$$
\mathrm{ASM}=\sum \sum(\mathrm{mhk})^{2}
$$

This is a measure of the uniformity of the gray level distribution of an image. When the numerical 
distribution of mhk is concentrated near the main diagonal, its corresponding ASM value is larger; otherwise, the ASM value is smaller.

$$
\mathrm{ENT}=-\sum \sum \mathrm{m}_{\mathrm{hk}} \operatorname{logm}_{\mathrm{hk}}
$$

When the $\mathrm{M}$ values in the gray co-occurrence matrix are different and dispersed, the ENT value is larger; otherwise, if the values of $\mathrm{m}$ and $\mathrm{k}$ are more concentrated, the ENT value is smaller.

\section{C. correlation formula}

$$
\mathrm{COR}=\frac{\sum_{K} \sum_{K} h k m_{A K}-\mu_{X} \mu_{Y}}{\sigma_{X} \sigma_{Y}}
$$

Four of these are $m_{x}, m_{y}$ mean and standard deviation, $m_{x}$ is the sum of each column element in the matrix $\mathrm{m}_{\mathrm{y}}$, and my is the sum of each row element in the matrix $\mathrm{m}$. The correlation quantity is used to describe the similarity between the elements of rows or columns in a matrix, and it is a measure of gray linear relations ${ }^{[3]}$.

The document uses this method to extract texture features, the effect is very good. The method has a long history and is also a human being an accepted method of texture analysis.

\section{Tamura Texture Representation}

Based on the study of human visual perception of texture, Tamura et al proposed the expression of texture features. Six properties of six components corresponding to Tamura texture features in view of psychology on texture features, respectively, roughness and contrast, line likeness, regularity and roughness. Among them, the first three components are especially important for image retrieval. Next, this paper focuses on the definition and mathematical expression of the three characteristics of roughness, contrast and orientation.

\section{A. roughness;}

Roughness is a measure of texture granularity, granularity is the basic particle size of texture, and the calculation of roughness can be divided into the following steps. First, calculate the average intensity of the pixels in the active window in the image size of $\left(2^{\mathrm{k}}+1\right) *\left(2^{\mathrm{k}}+1\right)$ pixels:

$$
\mathrm{A}_{\mathrm{K}}(\mathrm{x}, \mathrm{y})=\sum_{i=x-2^{k}}^{x+2^{k}-1} \sum_{j=y-2^{k}}^{y+2^{k}-1} \frac{g(i, j)}{\left(2^{k}+1\right)^{2}}
$$

Among them, $k=1, \ldots 6$, while $g(i, j)$ is the pixel intensity value located in (i, j). Then, for each pixel, the average intensity difference between the window in the horizontal and vertical directions is calculated separately.

$$
\begin{aligned}
& E_{k}(x, y)=\mid A_{k}\left(x+2^{k}, y\right)-A_{k}\left(x-2^{k}, y\right) \\
& E_{k v}(x, y)=\mid A_{k}\left(x, y+2^{k}\right)-A_{k}\left(x, y-2^{k}\right)
\end{aligned}
$$

For each pixel, the $\mathrm{K}$ value that makes the $\mathrm{E}$ value maximum (regardless of direction) is used to set the optimum size $S_{\text {best }}(x, y)=2^{k}$. Finally, the roughness can be obtained by calculating the average value of $S_{\text {best }}(x, y)$ in the whole image:

$$
\mathrm{F}_{\mathrm{CFS}}=\frac{1}{m \times n} \sum_{i=1}^{m} \sum_{j=1}^{n} \mathrm{~S}_{\text {best }}(\mathrm{i}, \mathrm{j})
$$

Another improvement of roughness features is to use histogram to describe the distribution of Sbest rather than simply calculate the average of Sbest as the above method. This improved roughness feature can express images or regions with many different texture features, so it is more favorable for image retrieval. This method is adopted in this paper.

\section{B. contrast}

Contrast refers to the distribution of image brightness, defined as:

$$
\text { Contrast }=\frac{\sigma}{\left(\alpha_{4}\right)^{\frac{1}{4}}}
$$

The right side of the equation is the bright 4 - center distance, and the $\mathrm{A}$ is the standard deviation. The formula is as follows: 


$$
\begin{gathered}
\sigma_{4}=\sum_{i=0}^{\text {range }} i^{4} \mathrm{p}(\mathrm{i}) \\
\text { mean }==\sum_{i=0}^{\text {range }} i \\
\sigma=\sqrt{\mu_{2}-\text { mean }^{2}}
\end{gathered}
$$

Range is the intensity range of the image brightness, and P (i) is the probability value of the $\mathrm{i}$ intensity.

\section{C. directionality;}

Directionality is a measure of the orientation of an image. First, the gradient and gradient directions of each pixel of the texture are calculated. Formula is as follows:

$$
\begin{gathered}
|\Delta G|=\frac{|\Delta H|+|\Delta V|}{2} \\
\theta=\tan ^{-1} \frac{\Delta V}{\Delta H}+\frac{\pi}{2}
\end{gathered}
$$

$\Delta \mathrm{H}$ and $\Delta \mathrm{V}$ respectively through the change of image convolution following two $3 \times 3$ operators from the horizontal and vertical direction of the amount.

When the gradient vectors of all pixels are computed, a histogram $h_{d}$ is constructed to represent $\theta$ values. Firstly, the range and range of $\theta$ are discretized, and then the number of pixels in each bin corresponding to the $G$ is larger than the given threshold. This histogram shows peak values for images with obvious directionality, and is smoother for images with no apparent orientation. Finally, the overall directionality of the image can be obtained by calculating the sharpness of the peaks in the histogram, which represents the following ${ }^{[4]}$ :

$$
\mathrm{F}_{\mathrm{dir}}=\sum \sum\left(\varphi-\varphi_{\mathrm{P}}\right){ }^{2} \mathrm{H}_{\mathrm{D}}(\varphi)
$$

The $\mathrm{P}$ in the upper representation represents the peak in the histogram, and $\mathrm{n}_{\mathrm{p}}$ is all the peaks in the histogram. For a peak $\mathrm{P}, \mathrm{w}_{\mathrm{p}}$ represents all of the bin contained in the peak, and $\varphi \mathrm{P}$ is the highest value bin.

The direction is measured at the width of the peak. If there are multiple peaks, find the width of each peak, then add up as the total width. These features are consistent with human perception and have been applied in many image retrieval systems.

Therefore, the Tamura texture includes roughness, contrast, and directionality. The following algorithms are presented for the implementation of roughness: first, the image is divided into $3 * 3$ blocks, and each of them is processed as follows:

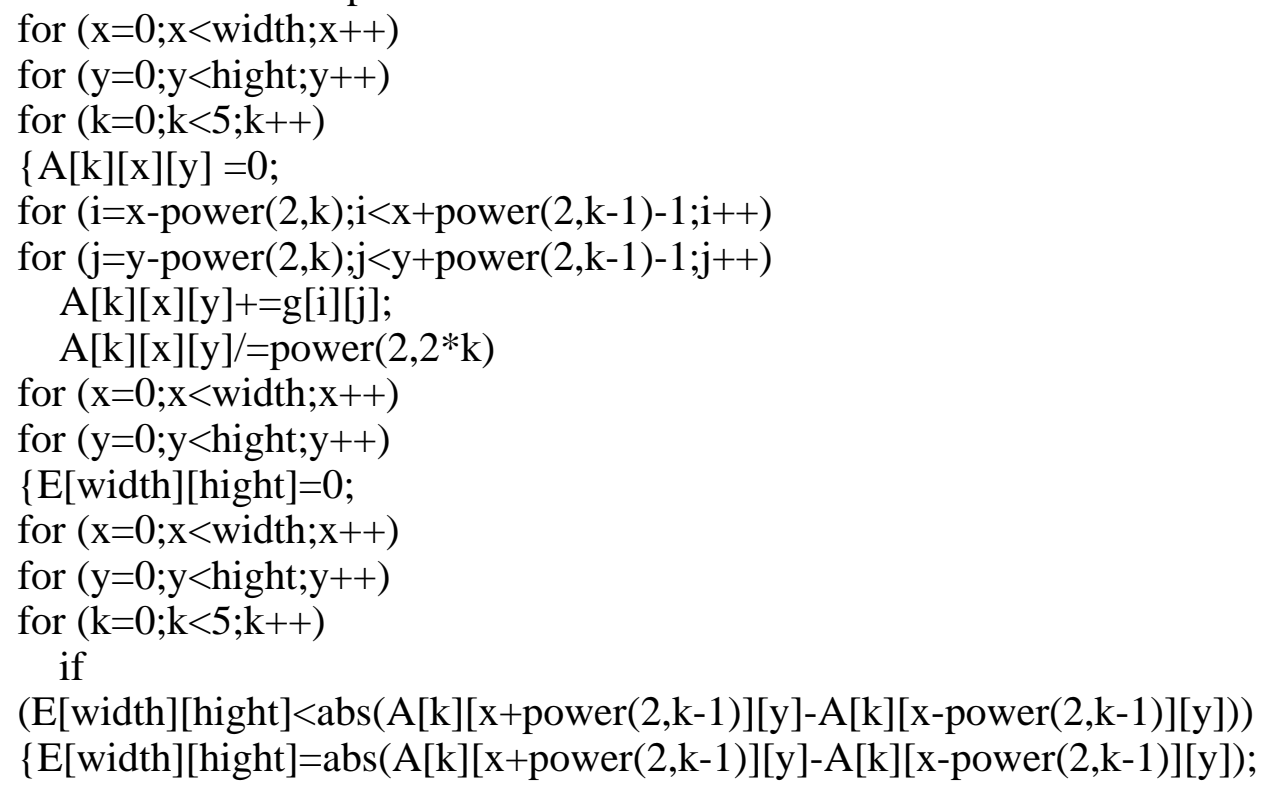




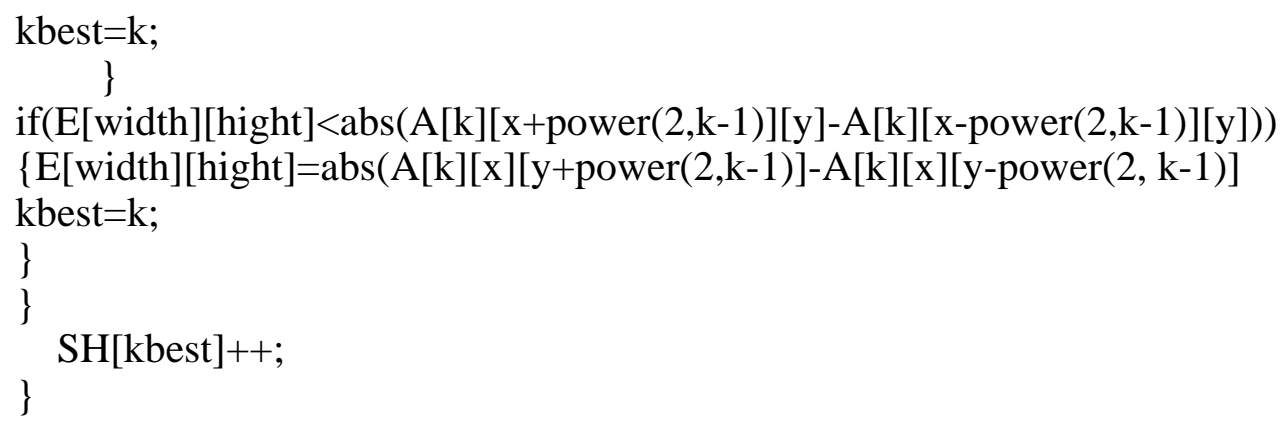

\section{Conclusions}

The texture in digital images is the spatial correlation of the gray or color of adjacent pixels, or the visual representation of the change of image gray level and color space position, which can be expressed as edges, shapes, stripes, color blocks and so on. Texture is an attribute of an image itself that reflects the roughness, smoothness, or regularity of an image. Detecting the similarity between images is an important method to judge whether the image is similar or not. Practice has shown that using texture for image retrieval is a very effective means.

Texture feature retrieval is based on the idea: to extract the texture features by the method of mathematics or information theory, the image grayscale or color space overall or each side with digital feature representation, then using texture analysis method to detect the texture element texton and access to relevant information distribution line ${ }^{[5]}$.

\section{References}

[1]Aalick R,Shanmugam K,Dinstein.Texture features for image classification,IEEE Transactions[J],Systems Man and Cybernetics,1973,3(6):17-20

[2] Li Xiancheng. Research on image retrieval technology based on main colors [J], traffic and computer, 2006, 24 (4): 8-10

[3] Manjunath B S.Texture Features for Browsing and Retrieval of Image Data[J], IEEE trans on image processing,1993,2(4):29-41

[4] Sun Junding, Xiao Wu. Image retrieval based on color distribution features [J], optoelectronics. Lasers, 2006,17 (8): 17-19

[5] Huang Yuanyuan, He Yunfeng. An image retrieval method based on color features [J] Chinese Journal of image and graphics, 2006,11 (12): 33-37 